\title{
Influence of Different Dates of Sowing, Fertilizer Level and Weedicides on Growth and Yield of Fenugreek (Trigonella foenum-graecum L.) under Semi-Arid Conditions
}

\author{
N.K. Meena*, S.S. Meena, Suman Gupta and G. Lal \\ ICAR-National Research Centre on Seed Spices, Ajmer (Rajasthan), India \\ *Corresponding author
}

A B S T R A C T

\section{Keywords \\ Sowing dates, Fertilizer doses, Weedicides, Trigonella foenum- graecum L., Semi- arid conditions \\ Article Info \\ Accepted: \\ 12 July 2018 \\ Available Online: \\ 10 August 2018}

An investigation was carried out at ICAR-National Research Centre on Seed Spices, Ajmer, Rajasthan during Rabi season 2016-17 to ascertain the suitable sowing date, fertilizers doses and weedicide for quality production of fenugreek variety AFg-3. Result illustrated that significantly higher plant height $(70.10 \mathrm{~cm})$, per plant primary branches (4.64), secondary branches (3.86), nodules (4.90), seed yield (1800 kg ha $\left.{ }^{-1}\right)$ and biological yield $\left(5755.55 \mathrm{~kg} \mathrm{ha}^{-1}\right)$ were recorded in $30^{\text {th }}$ October sown crop as compared to $15^{\text {th }}$ October and $15^{\text {th }}$ November sowing dates. Irrespective of sowing dates, the application of NPK level $\left(\mathrm{F}_{3}\right) 50: 50: 25 \mathrm{~kg} \mathrm{ha}^{-1}$ recoded highest plant height $(67.57 \mathrm{~cm})$, per plant primary branches (4.33), secondary branches (3.28), nodules (3.72), seed yield (1675.92 kg ha-1) and biological yield (5333.32 $\left.\mathrm{kg} \mathrm{ha}^{-1}\right)$. The pre-emergence application of oxadiargyl @ $75 \mathrm{~g}$ a.i. ha ${ }^{-1}$ provided more plant height $(65.60 \mathrm{~cm})$, per plant primary branches $(4.23)$, secondary branches (3.08), nodules (4.03), weed counts $\left(47.88\right.$ and 65.14 weeds $/ \mathrm{m}^{2}$ area at 45 and 90 DAS, respectively), seed yield (1643.22 $\mathrm{kg} \mathrm{ha}^{-1}$ ) and biological yield (5027.16 $\left.\mathrm{kg} \mathrm{ha}^{-1}\right)$. The combined effect of the treatments $\mathrm{D}_{2} \mathrm{xF}_{3} \mathrm{xW}_{2}$ given maximum plant height $(72.33 \mathrm{~cm})$, number of primary and secondary branches $(5.23$ and 4.33$)$, pods per plant (42.36), seed yield (2511.13 $\left.\mathrm{kg} \mathrm{ha}^{-1}\right)$, gross return (Rs.158934 ha-1), net return (109632 $\mathrm{ha}^{-1}$ ) and BCR (3.22).

\section{Introduction}

Fenugreek (Trigonella foenum-graecum L.) is an annual legume seed spice crop commonly known as methi, belongs to the family Fabaceae. The name fenugreek comes from the species name "foenum-graecum" means "Greek hay" (Flammang et al., 2004). It is a self-pollinated crop with chromosome no. $2 n=16$. It believed to be a native of an area extending from Iran to northern India (Marzougui et al., 2007) and cultivated worldwide. In India, it is cultivated mainly in Rajasthan, Gujarat, Madhya Pradesh, Chhattisgarh, Maharashtra, Haryana, Punjab and Uttar Pradesh in an area of 2.27 lakh hectare, produced 2.47 lakh tonnes of seeds with an annual productivity of $1085 \mathrm{~kg} / \mathrm{hectare}$ (Anonymous, 2016). Fenugreek is one of the major and important seed spice crop occupies 
a prime position throughout the globe to add taste and flavour in various food items. Fenugreek seeds contain carbohydrates (48\%), proteins $(25.5 \%)$, mucilaginous matter $(20 \%)$, fats $(7.9 \%)$, saponin $(4.8 \%)$ (Rao and Sharma, $1987)$ and volatile oil $(0.02 \%)$ (Ravindran et al., 2001). In England, seeds are extracted for steroidal substance "diosgenin" a sex hormone and oral contraceptive in family planning (Vasudevan et al., 2008). Fenugreek seeds contain 4-hydroxyisoleucine- a novel amino acid, which are known in traditional medicine for their anti-diabetic properties (Sauvaire et al., 1998). It significantly decreased the plasma triglyceride levels by $33 \%(\mathrm{P}<0.002)$, total cholesterol (TC) by $22 \%(\mathrm{P}<0.02)$, and free fatty acids by $14 \%$ (Narender et al., 2006). Fenugreek leaves and seeds are extensively used to prepare extract and powder for medicinal purpose (Basch et al., 2003). In addition to diabetic, seeds are also used for the treatment of various ailments i.e. chronic dysentery, diarrhoea, dyspepsia, chronic cough, enlargement of liver, spleen and rickets (Pruthi, 1979), stabilizing the insulin, blood sugar and haemoglobin levels (Mehta et al., 2010). Fenugreek seeds also contain hormone precursors that can increase milk production in nursing mothers and it is widely used for insufficient lactation.

In manufacturing industry, fenugreek extracts are used in soaps and different cosmetics. Steroid diosgenin used in pharmaceutical industry (Mehrafarin et al., 2010). It can be grown under wide range of climatic conditions. It requires cool climate and dry weather at the time of maturity. It can be grown in all types of soils, which is well drained. Unlike other legumes, it is fairly tolerant to salinity. However, it is better to avoid salty and acidic soils. Being a legume, its roots are endued with root nodules containing "Rhizobium" which fix atmospheric nitrogen for plant, thus its cultivation enriches the soil with nitrogen.
There is a need to standardize various agronomic techniques to improve seed yield and quality in fenugreek. Balanced nutrient, proper sowing time and managing weed population, obviously these three factors will not only enhance the productivity of seed but also decide the ultimate commercial success of fenugreek crop for pod vegetable. Similarly, the plants grown in last week of October to first week of November with proper nutrient management gives maximum vegetative growth of fenugreek in terms of plant height, number of branches per plant, number of leaves, number of pods, number of seeds per pod, weight of seeds per pod, seed yield per plant and seed yield per hectare (Nandre et al., 2011).

The growth and seed yield are largely influenced by the nutrient status of the soil apart from genetic potential of the variety. Altering the soil nutrients and fertility status by providing balanced and adequate dose of major nutrients like nitrogen, phosphorus and potassium as per the crop requirement, is one of the easiest way to boost up the productivity of fenugreek. It is important to standardize the optimum sowing times, major nutrients levels and effective weed management tactics for realizing better seed production and productivity of fenugreek crop. In the light of above cited facts and lack of above information on these aspects particularly for semi-arid conditions, the present study was undertaken to find out effect of sowing dates, NPK levels and weedicides on growth, yield and quality of fenugreek.

\section{Materials and Methods}

The field experiment entitled studies on effect of sowing dates, NPK levels and weedicides on the growth and yield of fenugreek (Trigonella foenum-graecum L.) was conducted during the Rabi season of 2016-17 at ICAR-National Research Centre on Seed 
Spices, Ajmer. Experimental site was located at $74^{0} 36^{\prime} 01^{\prime \prime}$ E longitude and $26^{\circ} 22^{\prime} 31^{\prime \prime} \mathrm{N}$ latitude with an altitude of $460.17 \mathrm{~m}$ above mean sea level. The region falls under $3^{\text {rd }}$ agro climatic zone of Rajasthan. The soil of research farm is sandy loam having $\mathrm{pH} 8$ to 8.3 and 0.15 to $0.23 \%$ organic carbon, available $\mathrm{N} 178.5 \mathrm{~kg} \mathrm{ha}^{-1}$ (low), $\mathrm{P}_{2} \mathrm{O}_{5} 12 \mathrm{~kg}$ $\mathrm{ha}^{-1}$ (medium), $\mathrm{K}_{2} \mathrm{O} 85 \mathrm{~kg} \mathrm{ha}^{-1}$ (low). The treatments consisted of three dates of sowing $\left(\mathrm{D}_{1}-15^{\text {th }}\right.$ October, $\mathrm{D}_{2}-30^{\text {th }}$ October and $\mathrm{D}_{3}-15^{\text {th }}$ November), three doses of fertilizers NPK levels $\left(F_{1}-30: 30: 15 \mathrm{~kg} / \mathrm{ha}, \mathrm{F}_{2}-40: 40: 20 \mathrm{~kg} / \mathrm{ha}\right.$ and $\mathrm{F}_{3}-50: 50: 25 \mathrm{~kg} / \mathrm{ha}$ ) and two weedicides (W $\mathrm{W}_{1}$-pendimethalin @ $1 \mathrm{~kg} / \mathrm{ha}$ and $\mathrm{W}_{2^{-}}$ oxadiargyl@75 g a.i./ha). The experiment was laid out in Randomized Block Design with factorial concept. Eighteen treatment combinations were applied in three replications. The fenugreek variety $\mathrm{AFg}-3$ was sown in standard crop geometry as accordance to package of practices adopted by ICARNRCSS. Full dose of phosphorus, potash and half dose of nitrogen were given as basal dose at the time of soil preparation for sowing. The remaining nitrogen was applied in two equivalent split doses as top dressing in standing crop in at 30 and 60 days after sowing (DAS). The NPK were applied in the form of fertilizer urea, di-ammonium phosphate (DAP) and murate of potash (MOP). The pre-calculated quantities of weedicides i.e. pendimethalin @ $1.0 \mathrm{~kg} / \mathrm{ha}$ and oxadiargyl@75 g a.i. /ha were applied with the help of knapsack sprayer just after sowing as pre-emergence weedicide.

\section{Data collection and statistical analysis}

Observations on plant height $(\mathrm{cm})$ were recorded from five randomly selected and tagged plants/plot at 30,60,90 DAS and at harvest measuring base of plant to tip of the main shoot. The numbers of primary and secondary branches per plant were recorded at 60, 90 DAS and at harvest. Fresh weight of plant $(\mathrm{g})$ and numbers of nodules per plant were recorded at 60 and 90 DAS, whereas, number of weeds per $\mathrm{m}^{2}$ area and their fresh (g) and dry weight (g) at 45 and 60 DAS along with days taken to 50 per cent flowering were recorded. The yield attributes viz., number of pods per plant, number of seeds per pod, pod length $(\mathrm{cm})$, pod weight $(\mathrm{g})$, test weight $(\mathrm{g})$, seed yield, biological yield and straw yield in $\mathrm{kg} / \mathrm{ha}$ and harvest index (\%) were recorded. All the data related to the growth parameters and yield and yield attributes were obtained and statistically analyzed using OPSTAT software developed by CCSHAU, Hisar (India).

\section{Results and Discussion}

\section{Growth parameters}

The data on various growth parameters viz., plant height $(\mathrm{cm})$, number of primary branches per plant, secondary branches per plant, number of nodules per plant at different growth stages and days taken to 50\% flowering was recorded and presented in table 1. It was illustrated that, the sowing dates, fertilizer doses and weedicide applications were significantly influenced all growth attributes. The higher plant height at 45, 60, 90 DAS and at harvest were obtained in crop sown on $30^{\text {th }}$ October with the application of NPK @ 50:50:25 kg/ha and oxadiargyl @ 75g a.i. /ha (pre-emergence). The maximum plant height $70.10,67.57$ and $65.60 \mathrm{~cm}$ was recorded in the treatment of $\mathrm{D}_{2}$ ( $30^{\text {th }}$ October), $\mathrm{F}_{3}$ (NPK level 50:50:25kg/ha) and $\mathrm{W}_{2}$ (oxadiargyl @ 75g a.i. /ha), respectively. However, non-significant response of both the weedicides on plant height was recorded at harvest but found better at early plant growth stages. Bhutia and Sharangi (2018) and Sultana et al., (2016) had recorded the similar kind of observations in fenugreek in respect to sowing dates; get support to the present findings. Dutta et al., (2008) had also found an 
increase in plant height in black cumin with the advancement of sowing times up to $15^{\text {th }}$ November. Numbers of primary and secondary branches per plant were also found highest in same sowing date ( $30^{\text {th }}$ October), fertilizer dose (NPK@ 50:50:25 kg/ha) and weedicide (oxadiargyl @ 75g a.i. /ha as preemergence) at harvesting stage. The above three treatments were found significantly superior over rest of the treatments. Similar trends in numbers of primary and secondary branches per plant in fenugreek were also recorded at 45, 60 and 90 DAS in all the treatments (Table1).

Number of nodules per plant at 60 and 90 DAS was differed significantly due to sowing dates irrespective of NPK levels and weedicides. The more nodules were recorded at 60 DAS as compared to 90 DAS in all treatments. At 60 DAS, 4.90 nodules/plant were found in crop sown $30^{\text {th }}$ October $\left(\mathrm{D}_{2}\right)$ as compared to $15^{\text {th }}$ October $\left(\mathrm{D}_{1}\right)(4.17)$ and $15^{\text {th }}$ November $\left(\mathrm{D}_{3}\right)$ (2.67). Similarly highest nodule (4.27 and 4.03 per plant) was found under fertilizer dose (NPK level 50:50:25 $\mathrm{kg} / \mathrm{ha}$ ) and weedicide (oxadiargyl @ 75g a.i. /ha.), respectively. Fresh weight of plant at 60 DAS and 90 DAS was influenced by sowing dates, NPK levels and weedicides. The higher fresh weight of plant $(29.36 \mathrm{~g})$ at 90 DAS was recorded in fenugreek crop sown on $30^{\text {th }}$ October followed by $15^{\text {th }}$ October $(26.98 \mathrm{~g})$ and $15^{\text {th }}$ November $(12.53 \mathrm{~g})$. The maximum fresh weight of plant $24.46 \mathrm{~g}$ and $24.13 \mathrm{~g}$ was found in fertilizers dose (NPK level 50:50:25 $\mathrm{kg} / \mathrm{ha}$ ) and weedicide (oxadiargyl @ 75g a.i. /ha.) at $90 \mathrm{DAS}$. The fenugreek crop sown on $30^{\text {th }}$ October had taken minimum days (51 days) to 50\% flowering with the application of fertilizers dose (NPK levels: 50:50:25 kg/ha) and weedicide (oxadiargyl @ 75g a.i. /ha) considered as most suitable date of sowing, fertilizers doses and weedicides for fenugreek under semi-arid conditions. The similar increase in growth parameters under higher fertilizer levels were also noticed by Kumar $e t$ al., (2004) in french bean and Mehta et al., (2010) in fenugreek. Similarly, the minimum number of weed counts $/ \mathrm{m}^{2}$ area viz., 47.88 and 65.14 were recorded in the plots treated with oxadiargyl @ 75g a.i. per hectare at 45 and 90 DAS, respectively (Table 2). Likewise fresh weight and dry weight of weed was obtained lower in same weedicide with the sowing date $30^{\text {th }}$ October and NPK level (30:30:15 kg/ha).

The data of combined effect of all treatments i.e. sowing dates, NPK levels and weedicides on growth attributes of fenugreek are presented in table 4, showed that the treatment combination of $\mathrm{D}_{2} \mathrm{xF}_{3} \mathrm{xW}_{1}$ given maximum average plant height $72.33 \mathrm{~cm}, 5.23$ primary, 4.33 secondary branches per plant, whereas highest number of nodules per plant (5.33) was recorded $\mathrm{D}_{1} \mathrm{xF}_{3} \times \mathrm{WW}_{2}$ treatment combination under semi-arid conditions.

\section{Yield attributes and yield}

The results obtained from the effect of different sowing dates, fertilizers doses (NPK level) and weedicides on yield attribute and yield of fenugreek are presented in table 3. It was revealed that the yield attribute i.e. number of pods/plant, pod length, pod weight and number of seed per pod were significantly influences with various sowing dates, NPK levels and weedicide use. The crop sown on $30^{\text {th }}$ October resulted maximum number of pods per plant (37.23), pod length $(9.95 \mathrm{~cm})$, pod weight $(9.19 \mathrm{~g})$, number of seeds per pod (15.98) and test weight (14.95 g) over early and late sown crops (Table 3). This might be due to better start and growth of fenugreek with favourable environmental and congenial micro-climate inside the soil, better embryo development and seed germination. These findings are in close conformity with the report made by Bhutia and Sharangi (2016) in fenugreek. Similarly, the maximum seed yield $(1800 \mathrm{~kg} / \mathrm{ha})$, straw yield $(3955.55 \mathrm{~kg} / \mathrm{ha})$ and biological yield $(5755.55 \mathrm{~kg} / \mathrm{ha})$ was obtained from $30^{\text {th }}$ October sown crop. 
Table.1 Effect of sowing dates, NPK levels and weedicides on various growth parameters at different plant growth stages of fenugreek under semi-arid conditions

\begin{tabular}{|c|c|c|c|c|c|c|c|c|c|c|c|c|c|c|c|}
\hline \multirow[t]{2}{*}{ Treatments } & \multicolumn{4}{|c|}{ Plant height $(\mathrm{cm})$} & \multicolumn{3}{|c|}{$\begin{array}{l}\text { No. of primary } \\
\text { branches/plant }\end{array}$} & \multicolumn{3}{|c|}{$\begin{array}{l}\text { No. of secondary } \\
\text { branches/plant }\end{array}$} & \multicolumn{2}{|c|}{$\begin{array}{l}\text { No. of } \\
\text { nodules } \\
\text { /plant }\end{array}$} & \multicolumn{2}{|c|}{$\begin{array}{l}\text { Fresh wt. of } \\
\text { plant (g) }\end{array}$} & \multirow[t]{2}{*}{$\begin{array}{c}\text { Days to } \\
50 \% \\
\text { flowering }\end{array}$} \\
\hline & $\begin{array}{c}45 \\
\text { DAS }\end{array}$ & $\begin{array}{c}\text { 60 } \\
\text { DAS }\end{array}$ & $\begin{array}{c}90 \\
\text { DAS }\end{array}$ & $\begin{array}{c}\text { At } \\
\text { harvest }\end{array}$ & $\begin{array}{c}60 \\
\text { DAS }\end{array}$ & $\begin{array}{c}90 \\
\text { DAS }\end{array}$ & $\begin{array}{c}\text { At } \\
\text { harvest }\end{array}$ & $\begin{array}{c}\text { 60 } \\
\text { DAS }\end{array}$ & $\begin{array}{c}90 \\
\text { DAS }\end{array}$ & $\begin{array}{c}\text { At } \\
\text { harvest }\end{array}$ & $\begin{array}{c}60 \\
\text { DAS }\end{array}$ & $\begin{array}{c}90 \\
\text { DAS }\end{array}$ & $\begin{array}{c}60 \\
\text { DAS }\end{array}$ & $\begin{array}{c}90 \\
\text { DAS }\end{array}$ & \\
\hline \multicolumn{16}{|l|}{ Sowing dates } \\
\hline $15^{\text {th }}$ October $\left(D_{1}\right)$ & 7.35 & 29.24 & 57.02 & 65.42 & 3.92 & 4.18 & 4.27 & 2.42 & 3.00 & 3.15 & 4.17 & 2.58 & 9.92 & 26.98 & 52.77 \\
\hline $30^{\text {th }}$ October $\left(\mathrm{D}_{2}\right)$ & 7.48 & 32.61 & 61.48 & 70.10 & 4.28 & 4.53 & 4.64 & 3.22 & 3.70 & 3.86 & 4.90 & 3.16 & 9.88 & 29.36 & 51.00 \\
\hline $15^{\text {th }}$ November $\left(D_{3}\right)$ & 5.37 & 29.05 & 54.69 & 60.12 & 2.57 & 3.10 & 3.38 & 1.01 & 1.88 & 2.05 & 2.67 & 2.38 & 4.46 & 12.53 & 60.83 \\
\hline SEm \pm & 0.14 & 0.72 & 0.68 & 0.84 & 0.07 & 0.07 & 0.08 & 0.08 & 0.09 & 0.06 & 0.10 & 0.09 & 0.50 & 0.87 & 0.58 \\
\hline $\mathrm{CD}(\mathrm{p}=\mathbf{0 . 0 5})$ & 0.41 & 2.08 & 1.96 & 2.43 & 0.20 & 0.22 & 0.24 & 0.23 & 0.26 & 0.19 & 0.31 & 0.25 & 1.43 & 2.51 & 1.67 \\
\hline \multicolumn{16}{|l|}{ NPK levels } \\
\hline $30: 30: 15 \mathrm{~kg} / \mathrm{ha}\left(\mathrm{F}_{1}\right)$ & 6.54 & 29.70 & 56.33 & 63.72 & 3.25 & 3.63 & 3.87 & 1.92 & 2.61 & 2.78 & 3.75 & 2.45 & 7.68 & 21.80 & 56.05 \\
\hline $40: 40: 20 \mathrm{~kg} / \mathrm{ha}\left(\mathrm{F}_{2}\right)$ & 6.61 & 30.46 & 55.82 & 64.33 & 3.63 & 3.95 & 4.08 & 2.16 & 2.82 & 3.00 & 3.72 & 2.65 & 7.48 & 22.61 & 54.77 \\
\hline $50: 50: 25 \mathrm{~kg} / \mathrm{ha}\left(\mathrm{F}_{3}\right)$ & 7.06 & 30.74 & 61.05 & 67.57 & 3.80 & 4.22 & 4.33 & 2.57 & 3.15 & 3.28 & 4.27 & 3.02 & 9.10 & 24.46 & 53.77 \\
\hline SEm \pm & 0.14 & 0.72 & 0.68 & 0.84 & 0.07 & 0.07 & 0.08 & 0.08 & 0.09 & 0.06 & 0.10 & 0.09 & 0.50 & 0.87 & 0.58 \\
\hline $\mathrm{CD}(\mathrm{p}=\mathbf{0 . 0 5})$ & 0.41 & NS & 1.96 & 2.43 & 0.20 & 0.21 & 0.24 & 0.23 & 0.26 & 0.19 & 0.31 & 0.25 & 1.50 & 2.62 & 1.67 \\
\hline \multicolumn{16}{|l|}{ Weedicides } \\
\hline $\begin{array}{l}\text { Pendimethalin @ } 1.0 \\
\text { kg/ha }\end{array}$ & 6.68 & 28.95 & 56.60 & 64.82 & 3.57 & 3.86 & 3.97 & 2.07 & 2.82 & 2.96 & 3.80 & 2.58 & 7.18 & 21.79 & 55.77 \\
\hline Oxadiargyl @ 75g a.i./ha & 6.78 & 31.65 & 58.87 & 65.60 & 3.62 & 4.01 & 4.23 & 2.36 & 2.90 & 3.08 & 4.03 & 2.84 & 9.00 & 24.13 & 53.96 \\
\hline SEm \pm & 0.11 & 0.59 & 0.55 & 0.69 & 0.05 & 0.06 & 0.07 & 0.06 & 0.07 & 0.05 & 0.08 & 0.07 & 0.40 & 0.71 & 0.47 \\
\hline $\mathrm{CD}(\mathrm{p}=\mathbf{0 . 0 5})$ & NS & 1.70 & 1.60 & NS & 0.17 & 0.12 & 0.20 & 0.56 & 0.22 & 0.17 & NS & 0.21 & 1.17 & 2.05 & 1.37 \\
\hline
\end{tabular}

*NPK: N- nitrogen, P-phosphorus and K-potash. 
Table.2 Effect of sowing dates, NPK levels and weedicides on number of weeds $/ \mathrm{m}^{2}$ area and fresh and dry weight of weeds $/ \mathrm{m} 2$ area in fenugreek under semi-arid conditions

\begin{tabular}{|c|c|c|c|c|c|c|}
\hline \multirow[t]{2}{*}{ Treatments } & \multicolumn{2}{|c|}{ No. of weed $/ \mathrm{m}^{2}$ area } & \multicolumn{2}{|c|}{ Fresh weight of weed (g) } & \multicolumn{2}{|c|}{ Dry weight of weed (g) } \\
\hline & 45 DAS & 90 DAS & 45 DAS & 90 DAS & 45 DAS & 90 DAS \\
\hline \multicolumn{7}{|l|}{ Sowing dates } \\
\hline $15^{\text {th }}$ October $\left(D_{1}\right)$ & 54.38 & 65.22 & 61.32 & 302.38 & 9.77 & 36.47 \\
\hline $30^{\text {th }}$ October $\left(D_{2}\right)$ & 53.61 & 62.94 & 60.91 & 236.58 & 9.55 & 31.57 \\
\hline $15^{\text {th }}$ November $\left(D_{3}\right)$ & 50.94 & 81.22 & 60.88 & 345.02 & 12.05 & 39.69 \\
\hline SEm \pm & 2.46 & 3.64 & 2.83 & 7.94 & 0.53 & 0.98 \\
\hline $\mathrm{CD}(\mathrm{P}=0.05)$ & NS & 10.48 & NS & 22.84 & 1.54 & 2.82 \\
\hline \multicolumn{7}{|l|}{ NPK levels } \\
\hline $30: 30: 15 \mathrm{~kg} / \mathrm{ha}\left(\mathrm{F}_{1}\right)$ & 45.38 & 73.44 & 65.53 & 306.72 & 11.58 & 38.88 \\
\hline 40:40:20 kg/ha $\left(F_{2}\right)$ & 58.77 & 61.55 & 50.36 & 232.72 & 8.02 & 31.76 \\
\hline $50: 50: 25 \mathrm{~kg} / \mathrm{ha}\left(\mathrm{F}_{3}\right)$ & 54.77 & 74.38 & 67.22 & 344.55 & 11.77 & 37.08 \\
\hline SEm \pm & 2.46 & 3.64 & 2.83 & 7.94 & 0.53 & 0.98 \\
\hline $\mathrm{CD}(\mathrm{P}=0.05)$ & 7.09 & 10.48 & 8.15 & 22.84 & 1.54 & 2.82 \\
\hline \multicolumn{7}{|l|}{ Weedicides } \\
\hline $\begin{array}{l}\text { Pendimethalin @ } 1.0 \text { kg/ha } \\
\left(W_{1}\right)\end{array}$ & 58.07 & 74.44 & 66.19 & 309.27 & 10.99 & 36.75 \\
\hline $\begin{array}{l}\text { Oxadiargyl @ 75g a.i./ha } \\
\left(\mathbf{W}_{2}\right)\end{array}$ & 47.88 & 65.14 & 55.88 & 280.05 & 9.92 & 35.06 \\
\hline SEm \pm & 2.01 & 2.97 & 2.31 & 6.48 & 0.43 & 0.80 \\
\hline $\mathrm{CD}(\mathrm{P}=0.05)$ & 5.79 & 8.55 & 6.66 & 18.64 & NS & NS \\
\hline
\end{tabular}

*NPK: N- nitrogen, P-phosphorus and K-potash. 
Table.3 Effect of different sowing dates, NPK levels and weedicides on yield attributes and seed yield of fenugreek under semi-arid conditions

\begin{tabular}{|c|c|c|c|c|c|c|c|c|c|}
\hline Treatments & $\begin{array}{c}\text { Number of } \\
\text { pod/plant }\end{array}$ & $\begin{array}{l}\text { Pod length } \\
\quad(\mathrm{cm})\end{array}$ & $\begin{array}{c}\text { Pod } \\
\text { weight }(g)\end{array}$ & $\begin{array}{l}\text { No. of } \\
\text { seed/pod }\end{array}$ & $\begin{array}{c}\text { Test } \\
\text { weight }(\mathrm{g})\end{array}$ & $\begin{array}{l}\text { Seed yield } \\
(\mathrm{kg} / \mathrm{ha})\end{array}$ & $\begin{array}{l}\text { Straw yield } \\
\text { (kg/ ha) }\end{array}$ & $\begin{array}{c}\text { Biological } \\
\text { yield }(\mathrm{kg} / \mathrm{ha})\end{array}$ & $\begin{array}{l}\text { Harvest Index } \\
(\%)\end{array}$ \\
\hline \multicolumn{10}{|l|}{ Sowing dates } \\
\hline $15^{\text {th }}$ October $\left(D_{1}\right)$ & 33.73 & 9.75 & 8.51 & 15.43 & 14.52 & 1661.11 & 3325.92 & 4987.03 & 33.98 \\
\hline $30^{\text {th }}$ October $\left(\mathrm{D}_{2}\right)$ & 37.23 & 9.95 & 9.19 & 15.98 & 14.95 & 1800.00 & 3955.55 & 5755.55 & 31.52 \\
\hline $15^{\text {th }}$ November $\left(D_{3}\right)$ & 25.50 & 9.69 & 6.10 & 15.91 & 12.51 & 1124.07 & 2501.85 & 3625.92 & 32.23 \\
\hline SEm \pm & 0.79 & 0.25 & 0.36 & 0.33 & 0.24 & 53.36 & 191.61 & 188.43 & 0.92 \\
\hline $\mathrm{CD}(\mathrm{P}=0.05)$ & 2.28 & NS & 1.03 & 1.01 & 0.70 & 153.40 & 550.85 & 541.70 & NS \\
\hline \multicolumn{10}{|l|}{ NPK levels } \\
\hline $30: 30: 15 \mathrm{~kg} / \mathrm{ha}\left(F_{1}\right)$ & 30.46 & 9.67 & 7.68 & 15.70 & 13.88 & 1440.75 & 2911.11 & 4351.85 & 33.71 \\
\hline 40:40:20 kg/ha $\left(F_{2}\right)$ & 31.43 & 9.78 & 8.00 & 15.50 & 13.99 & 1468.51 & 3214.81 & 4683.34 & 32.45 \\
\hline 50:50:25 kg/ha $\left(F_{3}\right)$ & 34.56 & 9.92 & 8.12 & 16.12 & 14.11 & 1675.92 & 3657.40 & 5333.32 & 31.57 \\
\hline SEm \pm & 0.79 & 0.25 & 0.36 & 0.33 & 0.24 & 53.36 & 191.61 & 188.43 & 0.920 \\
\hline $\mathrm{CD}(\mathrm{P}=0.05)$ & 2.28 & NS & 1.08 & 1.01 & NS & 153.40 & 550.85 & 541.70 & NS \\
\hline \multicolumn{10}{|l|}{ Weedicides } \\
\hline $\begin{array}{l}\text { Pendimethalin @ } 1.0 \mathrm{~kg} / \mathrm{ha} \\
\left(\mathrm{W}_{1}\right)\end{array}$ & 31.84 & 10.05 & 8.30 & 16.13 & 13.70 & 1413.57 & 3138.27 & 4551.85 & 32.22 \\
\hline Oxadiargyl @ 75g a.i./ha $\left(W_{2}\right)$ & 32.46 & 9.54 & 10.08 & 15.43 & 14.28 & 1643.22 & 3383.95 & 5027.16 & 32.94 \\
\hline $\mathrm{SEm} \pm$ & 0.64 & 0.20 & 0.29 & 0.27 & 0.20 & 43.57 & 331.88 & 153.85 & 0.75 \\
\hline $\mathrm{CD}(\mathrm{P}=0.05)$ & 1.94 & 0.62 & 0.78 & 0.82 & 0.57 & 125.25 & NS & 442.30 & NS \\
\hline
\end{tabular}

*NPK: N- nitrogen, P-phosphorus and K-potash 
Table.4 Interaction effect of different sowing dates, NPK levels and weedicides on growth parameters, yield attribute and seed yield of fenugreek

\begin{tabular}{|c|c|c|c|c|c|c|c|c|}
\hline Treatments & $\begin{array}{l}\text { Plant height } \\
(\mathrm{cm})\end{array}$ & $\begin{array}{l}\text { No. of primary } \\
\text { branches/plant }\end{array}$ & $\begin{array}{l}\text { No. of secondary } \\
\text { branches/plant }\end{array}$ & $\begin{array}{l}\text { No. of nodules } \\
\text { /plant }\end{array}$ & $\begin{array}{c}\text { No. of } \\
\text { pods/plant }\end{array}$ & $\begin{array}{l}\text { Pod length } \\
\quad(\mathrm{cm})\end{array}$ & $\begin{array}{c}\text { No. of } \\
\text { seeds/pod }\end{array}$ & $\begin{array}{l}\text { Seed yield } \\
(\mathrm{kg} / \mathrm{ha})\end{array}$ \\
\hline $\mathbf{D}_{1} \mathbf{F}_{1} \mathbf{W}_{1}$ & 66.13 & 3.86 & 3.06 & 3.83 & 30.60 & 9.93 & 15.13 & 1444.46 \\
\hline$D_{1} F_{1} W_{2}$ & 62.40 & 4.23 & 3.10 & 4.00 & 31.00 & 9.06 & 16.10 & 1400.00 \\
\hline $\mathbf{D}_{1} \mathrm{~F}_{2} \mathbf{W}_{1}$ & 65.03 & 4.03 & 2.73 & 3.53 & 31.40 & 8.80 & 14.43 & 1533.30 \\
\hline$D_{1} F_{2} W_{2}$ & 63.26 & 4.06 & 3.20 & 3.76 & 37.46 & 9.70 & 15.66 & 1866.66 \\
\hline$D_{1} F_{3} W_{1}$ & 65.53 & 4.30 & 3.63 & 4.60 & 36.93 & 10.96 & 16.10 & 1822.23 \\
\hline$D_{1} F_{3} W_{2}$ & 70.16 & 5.13 & 3.20 & 5.33 & 35.00 & 10.03 & 15.20 & 1900.00 \\
\hline $\mathrm{D}_{2} \mathrm{~F} 1 \mathrm{~W}_{1}$ & 67.06 & 4.16 & 3.06 & 4.46 & 38.13 & 10.26 & 17.00 & 1677.76 \\
\hline $\mathbf{D}_{2} \mathbf{F}_{1} \mathbf{W}_{2}$ & 68.26 & 4.23 & 3.23 & 5.06 & 39.70 & 9.70 & 15.56 & 2011.13 \\
\hline $\mathbf{D}_{2} \mathbf{F}_{2} \mathbf{W}_{1}$ & 70.50 & 5.23 & 4.06 & 4.86 & 33.46 & 10.63 & 16.66 & 1477.76 \\
\hline $\mathbf{D}_{2} \mathbf{F}_{2} \mathbf{W}_{2}$ & 70.43 & 4.26 & 4.20 & 4.86 & 31.06 & 9.46 & 14.80 & 1700.03 \\
\hline $\mathbf{D}_{2} \mathbf{F}_{3} \mathbf{W}_{1}$ & 72.00 & 4.73 & 4.26 & 4.96 & 38.66 & 10.20 & 16.80 & 1422.20 \\
\hline $\mathbf{D}_{2} \mathbf{F}_{3} \mathbf{W}_{2}$ & 72.33 & 5.23 & 4.33 & 5.20 & 42.36 & 9.43 & 15.06 & 2511.13 \\
\hline $\mathbf{D}_{3} \mathbf{F}_{1} \mathbf{W}_{1}$ & 56.83 & 3.36 & 2.10 & 2.46 & 21.40 & 9.83 & 15.86 & 1166.66 \\
\hline$D_{3} F_{1} W_{2}$ & 61.66 & 3.40 & 2.16 & 2.66 & 21.93 & 9.26 & 14.56 & 944.46 \\
\hline$D_{3} F_{2} W_{1}$ & 58.53 & 3.10 & 1.96 & 2.70 & 29.10 & 10.70 & 15.40 & 1033.33 \\
\hline$D_{3} F_{2} W_{2}$ & 58.26 & 3.80 & 1.83 & 2.63 & 26.13 & 9.43 & 16.06 & 1200.00 \\
\hline $\mathbf{D}_{3} \mathbf{F}_{3} \mathbf{W}_{1}$ & 61.80 & 2.93 & 1.80 & 2.76 & 26.90 & 9.16 & 17.76 & 1144.43 \\
\hline $\mathbf{D}_{3} \mathbf{F}_{3} \mathbf{W}_{2}$ & 63.63 & 3.70 & 2.46 & 2.80 & 27.53 & 9.76 & 15.83 & 1255.56 \\
\hline SEm \pm & 2.07 & 0.21 & 0.17 & 0.26 & 1.94 & 0.62 & 0.82 & 130.70 \\
\hline $\mathrm{CD}(\mathrm{P}=0.05)$ & NS & 0.63 & 0.48 & 0.78 & NS & 1.87 & NS & 375.76 \\
\hline
\end{tabular}

$\# \mathrm{D}_{1}-1^{\text {st }}$ sowing date $\left(15^{\text {th }}\right.$ October $), \mathrm{D}_{2}-2^{\text {nd }}$ sowing date $\left(30^{\text {th }}\right.$ October $), \mathrm{D} 3-3^{\text {rd }}$ sowing date $\left(15^{\text {th }}\right.$ November $) ; \mathrm{F}_{1}-(\mathrm{NPK}: 30: 30: 15 \mathrm{~kg} / \mathrm{ha}), \mathrm{F}_{2}-(\mathrm{NPK}: 40: 40: 20 \mathrm{~kg} / \mathrm{ha})$ and F3-(NPK:50:50:25 kg/ha) and $\mathrm{W}_{1}$ - pendimethalin @ 1.0 kg/ha and $\mathrm{W}_{2}$-oxadiargyl @ 75g a.i./ha. 
Table.5 Interaction effect of different sowing dates, NPK levels and weedicides on yield and economics of fenugreek under semi-arid conditions

\begin{tabular}{|c|c|c|c|c|c|c|}
\hline Treatment combinations & Seed yield & Straw yield & Gross returns & Cost of cultivation & Net returns & $\mathrm{B}: \mathrm{C}$ ratio \\
\hline $\mathbf{D}_{1} \mathbf{F}_{1} \mathbf{W}_{1}$ & 1444.46 & 2833.33 & 92334.69 & 46582.90 & 45751.79 & 1.98 \\
\hline$D_{1} F_{1} W_{2}$ & 1400.00 & 3266.66 & 90533.33 & 47395.40 & 43137.93 & 1.91 \\
\hline $\mathbf{D}_{1} \mathbf{F}_{2} \mathbf{W}_{1}$ & 1533.30 & 3322.23 & 98642.47 & 47743.60 & 50898.87 & 2.07 \\
\hline $\mathbf{D}_{1} \mathbf{F}_{2} \mathbf{W}_{2}$ & 1866.66 & 2933.33 & 117866.69 & 48556.10 & 69310.59 & 2.43 \\
\hline$D_{1} F_{3} W_{1}$ & 1822.23 & 3411.10 & 116156.18 & 48489.70 & 67666.48 & 2.40 \\
\hline$D_{1} F_{3} W_{2}$ & 1900.00 & 4188.90 & 122377.80 & 49302.00 & 73075.80 & 2.48 \\
\hline $\mathbf{D}_{2} \mathbf{F}_{1} \mathbf{W}_{1}$ & 1677.76 & 3344.43 & 107354.89 & 46582.90 & 60772.99 & 2.30 \\
\hline$D_{2} F_{1} W_{2}$ & 2011.13 & 3933.33 & 128534.65 & 47395.40 & 81139.25 & 2.71 \\
\hline $\mathbf{D}_{2} \mathbf{F}_{2} \mathbf{W}_{1}$ & 1477.76 & 3511.13 & 95688.29 & 47743.60 & 47944.69 & 2.00 \\
\hline $\mathbf{D}_{2} \mathbf{F}_{2} \mathbf{W}_{2}$ & 1700.03 & 4300.00 & 110601.98 & 48556.10 & 62045.88 & 2.28 \\
\hline $\mathbf{D}_{2} \mathbf{F}_{3} \mathbf{W}_{2}$ & 2511.13 & 4133.33 & 158934.71 & 49302.00 & 109632.71 & 3.22 \\
\hline $\mathbf{D}_{3} \mathbf{F}_{1} \mathbf{W}_{1}$ & 1166.66 & 1866.70 & 73733.42 & 46582.90 & 27150.52 & 1.58 \\
\hline$D_{3} F_{1} W_{2}$ & 944.46 & 2222.23 & 61112.49 & 47395.40 & 13717.09 & 1.29 \\
\hline $\mathrm{D}_{3} \mathrm{~F}_{2} \mathrm{~W} 1$ & 1033.33 & 2611.10 & 67222.2 & 47743.60 & 19478.58 & 1.41 \\
\hline $\mathbf{D}_{3} \mathbf{F}_{2} \mathbf{W}_{2}$ & 1200.00 & 2611.10 & 77222.20 & 48556.10 & 28666.10 & 1.59 \\
\hline$D_{3} F_{3} W_{1}$ & 1144.43 & 2833.33 & 74332.65 & 48489.70 & 25842.95 & 1.53 \\
\hline $\mathbf{D}_{3} \mathbf{F}_{3} \mathbf{W}_{2}$ & 1255.56 & 2866.66 & 81067.35 & 49302.00 & 31765.35 & 1.64 \\
\hline
\end{tabular}

$\# \mathrm{D}_{1}-1^{\text {st }}$ sowing date $\left(15^{\text {th }}\right.$ October $), \mathrm{D}_{2}-2^{\text {nd }}$ sowing date $\left(30^{\text {th }}\right.$ October $), \mathrm{D} 3-3^{\text {rd }}$ sowing date $\left(15^{\text {th }}\right.$ November $) ; \mathrm{F}_{1}-(\mathrm{NPK}: 30: 30: 15 \mathrm{~kg} / \mathrm{ha}), \mathrm{F}_{2}-(\mathrm{NPK}: 40: 40: 20 \mathrm{~kg} / \mathrm{ha})$ and F3-(NPK:50:50:25 kg/ha) and $\mathrm{W}_{1}$ - Pendimethalin @ 1.0 kg/ha and $\mathrm{W}_{2}$-oxadiargyl @ 75g a.i./ha. 
The significant variations due to fertilizer levels were recorded for yield and yield dynamics, i.e. maximum number of pods $\mathrm{m}$, (34.56), pod length $(9.92 \mathrm{~cm})$, pod weight/plant $(8.12 \mathrm{~g})$, number of seeds/pod (16.12) and seed yield $(1675.92 \mathrm{~kg} / \mathrm{ha})$ under the application of NPK level $\left(\mathrm{F}_{3}\right)$ 50:50:25 $\mathrm{kg} / \mathrm{ha}$ followed by NPK level $\left(\mathrm{F}_{2}\right)$ 40:40:20 NPK $\mathrm{kg} / \mathrm{ha}$ and 30:30:15 NPK $\mathrm{kg} / \mathrm{ha}\left(\mathrm{F}_{1}\right)$. Irrespective of sowing date and NPK levels, pre-emergence application of oxadiargyl @75 g a.i. per ha given best yield attributes and yield of fenugreek. The results are in accordance with the findings of Meena et al., (2013) and Maliwal and Gupta (1989).

The interaction effect of sowing dates, NPK levels and weedicides on yield attributes and yield of fenugreek illustrated that the treatment combination of $\mathrm{D}_{2} \mathrm{xF}_{3} \mathrm{xW}_{1}$ given maximum average number of pods/ plant (42.36) and seed yield $(2511.13 \mathrm{~kg} / \mathrm{ha})$. However, its response on pod length and number of seeds per pod was non-significant. Nandre et al., (2011) had also observed the comparable results in fenugreek are accordance with findings.

Economic analysis: Varying sowing dates, fertilizer doses and weedicides widely influenced gross return, net return as well as benefit cost ratio (BCR). The higher gross return (Rs. $158934 \mathrm{ha}^{-1}$ ), net return (Rs. 109632 $\mathrm{ha}^{-1}$ ) and BCR (3.22) were obtained in treatments combination of $\mathrm{D}_{2} \mathrm{XF}_{3} \mathrm{xW}_{2}$ (in $30^{\text {th }}$ October sowing date with NPK level 50:50:25 $\mathrm{kg} / \mathrm{ha}$ and pre-emergence use of oxadiargyl@ $75 \mathrm{~g}$ a.i. /ha) followed by D2xF1xW2 (Table 5). The higher return in $30^{\text {th }}$ October sown crop with NPK level 50:50:25 kg/ha and oxadiargyl @ $75 \mathrm{~g}$ a.i./ha is due to higher plant growth, number of pods/ plant and seed yield. The similar observations were also recorded by Nandre et al., (2011) and Bhutia and Sharangi (2016) are close conformity of present findings. In conclusion, it was evident from the study that, the fenugreek variety AFg-3 sown on $30^{\text {th }}$ October and applied fertilizers doses NPK $50: 50: 25 \mathrm{~kg} / \mathrm{ha}$ with pre-emergence application of oxadiargyl @ $75 \mathrm{~g}$ a.i./ha is better for realizing higher growth attributes, seed yield, net return and BCR among all the treatment combinations.

\section{References}

Anonymous (2016). Statistics of spice's area, production and productivity in India. Directorate of Arecanut and Spice Development, Calicut, Kerala.

Basch, E., Ulbricht, C., Kuo, G., Szapary, P. and Smith, M. (2003). Therapeutic applications of fenugreek. Alt. Med. Rev., 8: 20-27.

Bhutia, P.H. and Sharangi, A.B. (2016). Effect of dates of sowing and soil moisture level in different growth stages and yield dynamics of fenugreek (Trigonella foenum-graecum L.) National Acadamy Science Lett., 39 (2): 77-80.

Bhutia, P.H. and Sharangi, A.B. (2018) Influence of date of sowing and irrigation scheduling on phenology, growth and yield dynamics of fenugreek (Trigonella foenum-graecum L.), Legume Research, 41 (2): 275-280.

Dutta, D., Bandyopadhyay, P. and Maiti, D. (2008). Effect of $\mathrm{P}$ fertilization and growth regulators on yield, nutrient uptake and economics of fenugreek (Trigonella foenum-graecum L.). Research on Crops, 9 (3): 599-601.

Flammang, A.M., Cifone, M.A., Erexson, G.L. and Stankowski, L.F. (2004). Genotoxicity testing of a fenugreek extract. Food and Chemical Toxicology, 42:1769-1775.

Kumar, M., Sinha, K.K. and Roysherma, R.P. (2004). Effect of organic manure, NPK and boron application on the productivity of french bean in sandy loam soil of north Bihar. Indian J. Pulse Res., 17:42-44.

Maliwal, P.L. and Gupta, O.P. (1989). Study on the effect of four herbicides with and without applied phosphorus on weed control and seed yield of fenugreek (Trigonella foenum-graecum L.), Tropical Pest Management, 35 (3): $307-$ 310. 
Marzougui, N., Ferchichi, A., Guasmi, F. and Beji, M. (2007). Morphological and chemical diversity among 38 tunisian cultivars of (Trigonella foenumgraecum L.). J. Food Agric. Environ, $5: 245-250$

Meena, S.S., Mehta, R.S., Lal, G. and Anwer, M.M. (2013). Economic feasibility of weed management practices in fenugreek. Indian Journal Horticulture, 70 (1): 150-153.

Mehrafarin, A., Qaderi, A., Rezazadeh, S., Naghdi, B.H., Noormohammadi G.H. and Zand E. (2010). Bioengineering of important secondary metabolites and metabolic pathways in fenugreek (Trigonella foenum-graecum L.). J. of Medicinal Plants, 9 (35): 1-18.

Mehta, R.S., Patel, B.S, Meena, S.S. and Meena, R.S. (2010). Influence of nitrogen, phosphorus and bio-fertilizers on growth characters and yield of fenugreek (Trigonella foenum-graecum L.). Journal of Spices and Aromatic Crops, 19 (1\&2): 23-28.

Mehta, R.S., Patel, B.S., Singh, R.K., Meena, S.S. and Malhotra, S.K. (2010). Growth and yield of fenugreek (Trigonella foenum-graecum L.) as influenced by irrigation levels and weed management practices. Journal of Spices and Aromatic Crops. 19:14-22.

Nandre, D.R., Ghadge, R.G. and Rajput, B.S. (2011). Effect of sowing dates and nutrient management on growth and seed yield of fenugreek. Advance Research Journal Crop Improvement, 2
(2): 215-220.

Narender, T., Puri, A., Shweta, Khaliq, T., Saxena, R., Bhatia, G. and Chandra, R. (2006). 4-hydroxyisoleucine an unusual amino acid as antidyslipidemic and antihyperglycemic agent. Bioorg Med Chem Lett., 16 (2):293-306.

Pruthi, J.S. (1979). Spice and condiment, National Book Trust, 108-111.

Rao, P.U. and Sharma, R.D. (1987). An evaluation of protein quality in fenugreek seed and their supplementary effect. Food Chemistry, 24:1-9.

Ravindran, P.N., Kallupurachal, J.A. and Sivaranam, N. (2001). AICRP on spices status of seed spice research. Indian Journal Arecanut Spices and Medicinal Plants, 3 (3): 97-110.

Sauvaire, Y., Petit, P., Broca, C., Manteghetti, M., Baissac, Y., Fernandez-Alvarez, J., Gross, R., Roye, M., Leconte, A., Gomis, R. and Ribes, G. (1998). 4hydroxyisoleucine: a novel amino acid potentiator of insulin secretion. Diabetes, 47(2):206-210.

Sultana, S., Das, G., Das, B. and Sarkar, S. (2016). Influence of date of sowing on growth and yield dynamics of fenugreek. International Journal of Green Pharmacy, 10 (4): 234.

Vasudevan, S.N., Sudarshan, J.S., Kurdikeri, M.B. and Dharmatti, P.R. (2008). Influence of pinching of apical bud and chemical sprays on seed yield and quality of fenugreek. Karnataka Journal Agriculture Science. 21 (1): 26-29.

\section{How to cite this article:}

Meena, N.K., Meena, S.S., Suman Gupta and Lal, G. 2018. Influence of Different Dates of Sowing, Fertilizer Level and Weedicides on Growth and Yield of Fenugreek (Trigonella foenum-graecum L.) under Semi-Arid Conditions. Int.J.Curr.Microbiol.App.Sci. 7(08): 1844-1854. doi: https://doi.org/10.20546/ijcmas.2018.708.212 